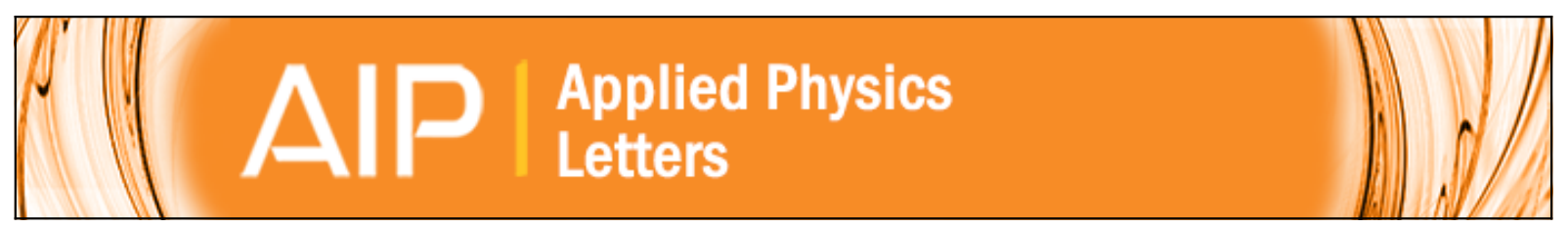

\title{
Photocurrent-voltage relation of resonant tunneling diode photodetectors
}

Andreas Pfenning, Fabian Hartmann, Mariama Rebello Sousa Dias, Fabian Langer, Martin Kamp, Leonardo

Kleber Castelano, Victor Lopez-Richard, Gilmar Eugenio Marques, Sven Höfling, and Lukas Worschech

Citation: Applied Physics Letters 107, 081104 (2015); doi: 10.1063/1.4929424

View online: http://dx.doi.org/10.1063/1.4929424

View Table of Contents: http://scitation.aip.org/content/aip/journal/apl/107/8?ver=pdfcov

Published by the AIP Publishing

\section{Articles you may be interested in}

Cavity-enhanced resonant tunneling photodetector at telecommunication wavelengths

Appl. Phys. Lett. 104, 101109 (2014); 10.1063/1.4868429

Photocurrent spectroscopy of intersubband transitions in GalnAsN/(Al)GaAs asymmetric quantum well infrared photodetectors

J. Appl. Phys. 112, 084502 (2012); 10.1063/1.4754573

One $\mathrm{THz}$ harmonic oscillation of resonant tunneling diodes

Appl. Phys. Lett. 87, 233501 (2005); 10.1063/1.2139850

Resonant two-photon photoemission in quantum-well infrared photodetectors

Appl. Phys. Lett. 84, 5162 (2004); 10.1063/1.1763978

Quantitative simulation of a resonant tunneling diode

J. Appl. Phys. 81, 3207 (1997); 10.1063/1.364151

\section{AIP $\left.\right|_{\text {APL Photonics }}$}

APL Photonics is pleased to announce Benjamin Eggleton as its Editor-in-Chief

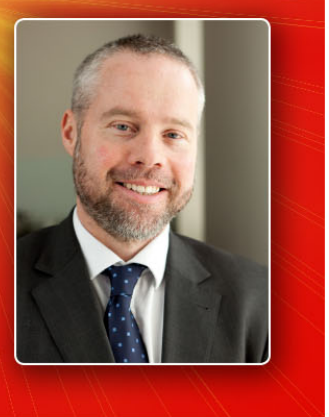




\title{
Photocurrent-voltage relation of resonant tunneling diode photodetectors
}

\author{
Andreas Pfenning, ${ }^{1, a)}$ Fabian Hartmann, ${ }^{1}$ Mariama Rebello Sousa Dias, ${ }^{2}$ Fabian Langer, ${ }^{1}$ \\ Martin Kamp, ${ }^{1}$ Leonardo Kleber Castelano, ${ }^{2}$ Victor Lopez-Richard, ${ }^{2}$ \\ Gilmar Eugenio Marques, ${ }^{2}$ Sven Höfling, ${ }^{1,3}$ and Lukas Worschech ${ }^{1}$ \\ ${ }^{1}$ Technische Physik and Wilhelm Conrad Röntgen Research Center for Complex Material Systems, \\ Physikalisches Institut, Universität Würzburg, Am Hubland, D-97074 Würzburg, Germany \\ ${ }^{2}$ Departamento de Fisica, Universidade Federal de Sao Carlos, 13565-905 Sao Carlos, SP, Brazil \\ ${ }^{3}$ SUPA, School of Physics and Astronomy, University of St. Andrews, St. Andrews, KY16 9SS, United Kingdom
}

(Received 24 June 2015; accepted 10 August 2015; published online 24 August 2015)

We have investigated photodetectors based on an $\mathrm{AlGaAs} / \mathrm{GaAs}$ double barrier structure with a nearby lattice-matched GaInNAs absorption layer. Photons with the telecommunication wavelength $\lambda=1.3 \mu \mathrm{m}$ lead to hole accumulation close to the double barrier inducing a voltage shift $\Delta V(V)$ of the current-voltage curve, which depends strongly on the bias voltage $V$. A model is proposed describing $\Delta V(V)$ and the photocurrent response in excellent agreement with the experimental observations. According to the model, an interplay of the resonant tunneling diode (RTD) quantum efficiency $\eta(V)$, the lifetime of photogenerated and accumulated charge carriers $\tau(V)$, and the RTD current-voltage relation in the dark determines best working parameters of RTD photodetectors. Limitations and voltage dependencies of the photoresponse are discussed. (C) 2015 AIP Publishing LLC. [http://dx.doi.org/10.1063/1.4929424]

Resonant tunneling diodes (RTDs) are nonlinear devices with a large spectrum for applications, ranging from high frequency oscillators up to the THz range, logic elements, nanothermometry, and high sensitive light detectors at the telecommunication wavelength. ${ }^{1-7}$ The broad spectrum of applications is due to the RTD's distinguished characteristics, e.g., the region of negative differential conductance, and an inherent high speed of electron dynamics combined with a relative structural simplicity. ${ }^{8}$ RTDs operated as photodetectors show a remarkable photoresponse with the possibility to detect single photons. ${ }^{9-13}$ Hereby, the RTD is serving as an internal amplifier of weak electric signals caused by photogenerated charge carriers. Extensive work has been done, e.g., to demonstrate single photon detection and even photon counting, $, 913,14$ yet some dynamic effects that shape the RTD photocurrentvoltage relation itself have remained uncharacterized. As demonstrated here, the photocurrent response is built by the intertwining of various processes of different nature.

Charge accumulation of photogenerated holes at the resonant tunneling structure (RTS) has been identified as reason for the RTD photosensitivity. ${ }^{15}$ Coelho et al. have provided a model of the RTD current-voltage characteristic under illumination by calculating a photoinduced voltage shift and integrating it into Schulman's equation. ${ }^{16}$ The voltage shift is given as a function of accumulated holes and is assumed constant across the whole bias voltage range. However, in our study, the photo-induced voltage shift is found to be strongly dependent on the bias voltage. Thus, to exploit the RTD photodetector's full potential, the best working points must be identified. This requires detailed knowledge of the RTD's photocurrent-voltage relation and the different parameters, which define it.

Here, we present a general study on the photoresponse and charge accumulation dynamics of RTD photodetectors.

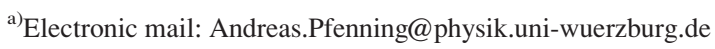

The studied RTD is composed of an AlGaAs/GaAs double barrier resonant tunneling structure with a nearby and latticematched GaInNAs absorption layer. The device was studied for light sensing at the telecommunication wavelength at $\lambda=1.3 \mu \mathrm{m}$ by means of electro-optical transport measurements. A strong nonlinear photocurrent-voltage relation was found and is attributed to various voltage dependent parameters. For low voltages, the photocurrent is negligibly small, mainly due to a low quantum efficiency $\eta(V)$. For high voltages the photoresponse is limited by the mean lifetime $\tau(V)$ of photogenerated holes that are confined in the vicinity of the RTS.

Fig. 1(a) shows a three dimensional sketch of the RTD photodetector. The RTD was grown by molecular beam epitaxy. The RTD's double barrier resonant tunneling structure consists of two $3 \mathrm{~nm}$ wide $\mathrm{Al}_{0.6} \mathrm{Ga}_{0.4}$ As barriers (red) embedding a $4 \mathrm{~nm}$ wide GaAs quantum well (grey). Between second AlGaAs barrier and GaInNAs absorption layer (light blue), a GaAs spacer layer with thickness of $1 \mathrm{~nm}$ was grown. The spacer layer is necessary to reduce defects at the RTS. ${ }^{17}$ The $\mathrm{Ga}_{0.89} \mathrm{In}_{0.11} \mathrm{~N}_{0.04} \mathrm{As}_{0.96}$ absorption layer was grown lattice-matched to GaAs with a bandgap energy of $E_{\text {gap }} \cong 0.95 \mathrm{eV}$ and thickness of $158 \mathrm{~nm}$. The first $14 \mathrm{~nm}$ were undoped, while subsequently $144 \mathrm{~nm}$ were Si-doped with $n=1.0 \times 10^{17} \mathrm{~cm}^{-3}$. RTD mesas with a diameter of $5 \mu \mathrm{m}$ were fabricated using standard lithographic and dry chemical etching techniques. The bottom contact (substrate) was formed by an alloyed $\mathrm{Ni} / \mathrm{AuGe} / \mathrm{Ni} / \mathrm{Au}$ contact. On top, a $\mathrm{Ti} / \mathrm{Pt} / \mathrm{Au}$ ring-shaped contact was evaporated, providing optical access combined with good electric contact. A polymer (Benzocyclobutene (BCB)) was used for mesa isolation.

In Fig. 1(b), the schematic conduction (CB) and valence (VB) band profiles under an applied bias voltage are shown. In the biased RTD photodetector, photogenerated holes $\left(\mathrm{h}^{+}\right)$ can accumulate at the RTS, which in turn enhances the local electrical field and causes an additional voltage drop across 

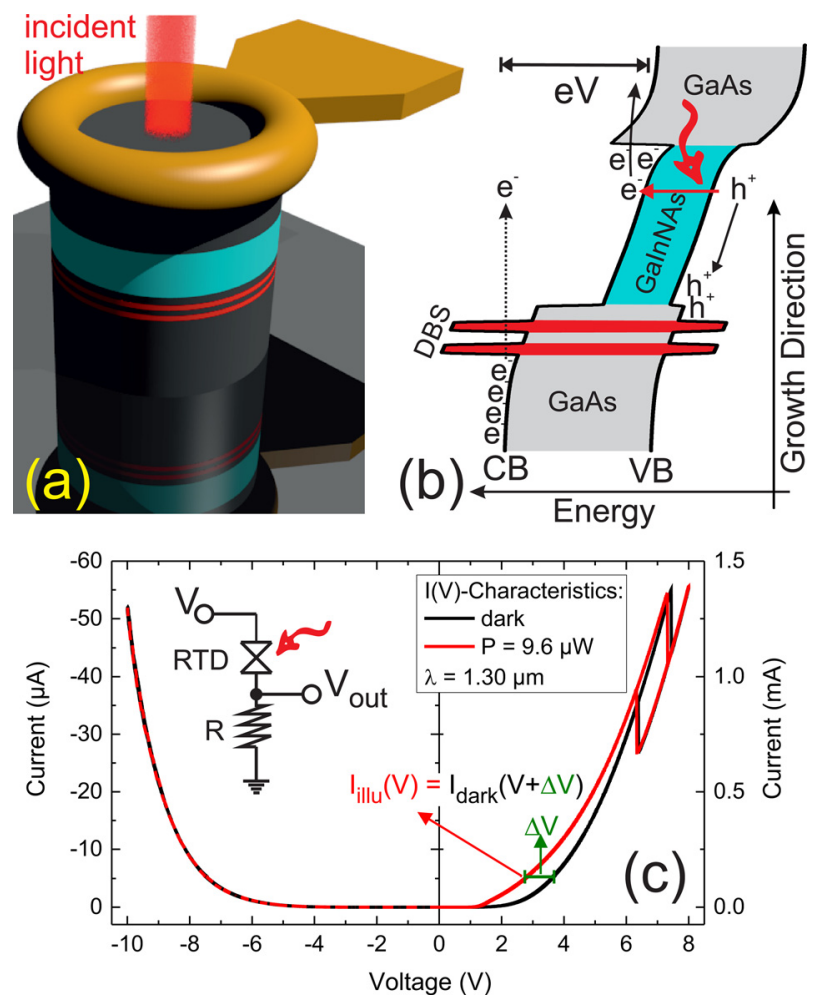

FIG. 1. (a) Three dimensional device layout of the RTD mesa structure with $\mathrm{Au}$ ring contact for electrical and optical access. The RTD heterostructure is color-coded. Red represents the AlGaAs barriers, light blue indicates the GaInNAs absorption layer, and GaAs is grey. (b) Sketch of the conduction band $(\mathrm{CB})$ and valence band (VB) profile under an applied bias voltage. (c) I(V)-characteristic in the dark (black) and under illumination (red) with $P=9.6 \mu \mathrm{W}$. The asymmetry of the $\mathrm{I}(\mathrm{V})$-characteristic is due to the asymmetric heterostructure layout. When illuminated and for positive bias voltages, the $\mathrm{I}(\mathrm{V})$-characteristic shifts towards smaller voltages due to accumulation of photogenerated holes at the resonant tunneling structure. The inset shows a sketch of the electrical circuit.

the RTS. ${ }^{15,16,18}$ As a result, the current-voltage (I(V)-) characteristic under illumination is shifted towards lower bias voltages (see Fig. 1(c)).

The inset in Fig. 1(c) shows a sketch of the electrical circuit. The RTD was operated in a two-terminal setup (with a single bottom contact), where a DC bias voltage $V$ was applied to the Au-ring contact by a high precision voltage source (Hewlett-Packard 3251 Universal Source), and the current was measured as voltage drop $V_{\text {out }}$ across a series resistor with a digital multimeter (Keithley 2000 Multimeter). $\mathrm{I}(\mathrm{V})$-characteristics were conducted in the dark and under illumination. A laser with emission wavelength $\lambda=1.30 \mu \mathrm{m}$ was used for excitation. The incident illumination power was kept constant at $P=9.6 \mu \mathrm{W}$. All measurements were performed at room temperature. Dark and illuminated I(V)characteristics are shown in Fig. 1(c) as black and red lines, respectively. The $\mathrm{I}(\mathrm{V})$-characteristic is asymmetric, due to the asymmetric heterostructure. For positive values of $V$, the current grows exponentially, and a pronounced bistability with threshold voltages $V_{t 1}=7.43 \mathrm{~V}$ (up-sweep direction) and $V_{t 2}=6.72 \mathrm{~V}$ (down-sweep direction) was found. For negative bias voltage, the current is diminished compared to positive bias voltage, and no region of negative differential conductance was found. When illuminated, the positive I(V)-characteristic (red line) shifts to smaller voltages by a photoinduced voltage shift $\Delta V$, caused by an accumulation of photogenerated holes near the RTS. For negative bias, no photosensitivity was observed, demonstrating that no accumulation of photogenerated holes occurs.

The photocurrent $I_{P h}$ was measured by subtracting the current in the dark $I_{\text {dark }}$ from the current under illumination $I_{i l l u}$. The resulting photocurrent-voltage relation $I_{P h}(V)$ is depicted in Fig. 2(a). $I_{P h}(V)$ is an asymmetric and nonlinear function of $V$, with a maximum of $120 \mu \mathrm{A}$ at $V=4.2 \mathrm{~V}$. The two negative spikes are due to threshold voltage shifts of $V_{t 1}$ and $V_{t 2}$. No photocurrent was observed for bias voltages below $0.35 \mathrm{~V}$. Since $I_{\text {illu }}(V)=I_{\text {dark }}(V+\Delta V),{ }^{16}$ the photocurrent-voltage relation can be defined as

$$
I_{P h}(V)=I_{\text {dark }}(V+\Delta V)-I_{\text {dark }}(V) .
$$

Thus, the asymmetric and nonlinear photocurrentvoltage relation is ascribed to a voltage-dependent $\Delta V(V)$. Fig. 2(a) shows $\Delta V$ versus bias voltage. $\Delta V(V)$ is an asymmetric and nonlinear function of $V$, with a maximum of $0.99 \mathrm{~V}$ at $V=1.8 \mathrm{~V}$. Elsewhere, $\Delta V$ declines and approaches zero. $\Delta V$ is determined by the number $N_{h}$ of photogenerated holes accumulated at the RTS times the additional voltage drop $V_{h}$ across the RTS caused by a single accumulated hole divided by the RTD leverage factor $n_{R T D} 16$
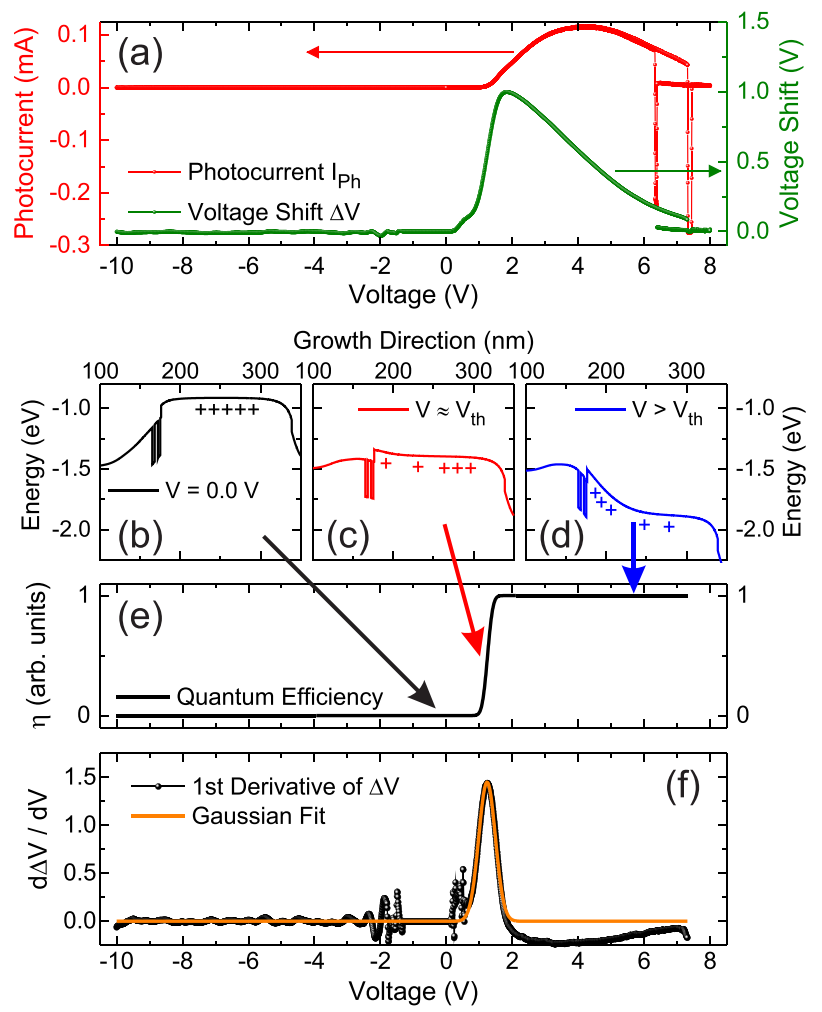

FIG. 2. (a) Photocurrent-voltage relation $I_{P h}(V)$ and photoinduced voltage shift $\Delta V(V)$. The photocurrent for negative bias is negligibly small. For positive bias, the photocurrent displays a maximum of $0.12 \mathrm{~mA}$ at $4.2 \mathrm{~V}$, and two negative spikes. (b)-(d) Simulations of the valence band profile for increasing bias voltages. A critical bias voltage must be surpassed to ensure efficient capturing of photogenerated holes at the resonant tunneling structure. (e) Sketch of the RTD quantum efficiency. (f) First derivative of the photoinduced voltage shift $\Delta V(V)$. The Gaussian component confirms that the quantum efficiency can be modeled by an error function. 


$$
\Delta V=N_{h} \times \frac{V_{h}}{n_{R T D}} .
$$

Here, $n_{R T D}$ and $V_{h}$ are constants defined by material parameters and heterostructure design. While $n_{R T D}$ can be extracted from the $\mathrm{I}(\mathrm{V})$-characteristic in the dark to be $n_{R T D}$ $=(2.6 \pm 0.3) \%, V_{h}$ is obtained by a simple electrostatic calculation to be $V_{h}=(0.89 \pm 0.05) \mu \mathrm{V} .^{16}$ The accumulated holes' dynamics can be characterized by their generation and escape rates included in the second and first terms, at the right side of the equation

$$
\frac{d N_{h}}{d t}=-\frac{N_{h}}{\tau(V)}+\eta(V) \times \frac{P}{h \nu},
$$

where $\tau(V)$ is the lifetime of accumulated holes at the RTS, $P / h \nu$ is the incident photon flux (given by the incident illumination power $P$ divided by the photon energy $h \nu$ ), and $\eta(V)$ is the RTD quantum efficiency. The RTD quantum efficiency is an external quantum efficiency defined as the probability of an incident photon to generate a minority charge carrier (hole), which is captured and accumulated at the RTS. For cw-illumination, an equilibrium condition $\left(d N_{h} / d t=0\right)$ is reached and thus

$$
\Delta \mathrm{V}(\mathrm{V})=\eta(V) \times \tau(V) \times \frac{P}{h \nu} \times \frac{V_{h}}{n_{R T D}}
$$

For negative bias, the applied electric field forces photogenerated holes away from the RTS, hence $\eta \rightarrow 0$. In Figs. 2(b)-2(d), simulations of the valence band profile are plotted for increasing bias voltages from (b) to (d). Due to a built-in electric field, a critical voltage $V_{t h, \eta}$ must be surpassed to ensure efficient capturing of photogenerated holes for accumulation at the RTS. We define $V_{t h, \eta}$ as the bias voltage, at which half the maximum quantum efficiency is reached. From first principles, $\eta(V)$ is assumed to be a step-function. The calculated quantum efficiency as shown in Fig. 2(e) indicates that a simple drift-diffusion model describes the RTD quantum efficiency well. ${ }^{19}$ To determine the step-like function, the first derivative of $\Delta V(V)$ was taken and is shown in Fig. 2(f). The Gaussian shaped peak confirms that $\eta(V)$ can be modeled by an error-function

$$
\eta(V)=1 / 2 \times \eta_{0}\left(1+\operatorname{erf}\left(\gamma\left(V-V_{t h, \eta}\right)\right)\right)
$$

where $\gamma$ is a parameter that accounts for steepness of the transition. For large enough voltages, $\eta(V)$ approaches a constant value $\eta_{0}$, which accounts for the absorption processes within the GaInNAs layer and loss mechanisms, such as reflections at the semiconductor-air interface. For our sample, we have calculated a value of $\eta_{0}=5.2 \%$, taking only absorption within the GaInNAs layer and reflection at the semiconductor-air interface into account. ${ }^{20}$ Besides the nonlinear, quantum efficiency $\eta(V)$ that acts as the limiting factor of the RTD photoresponse for small and negative bias voltages, one has to investigate the voltage dependence of the mean lifetime $\tau(V)$ of captured and accumulated holes.

To characterize the relevant lifetime, the RTD was excited with weak rectangular laser pulses of $100 \mu$ s width, and the photocurrent was measured as a function of time and bias voltages from $V=0.00 \mathrm{~V}$ up to $V=3.20 \mathrm{~V}$. The illumination power was set constant at $P=110 \mathrm{pW}$. The timeresolved photocurrent measurements were conducted using a digital oscilloscope instead of the Keithley 2000 Digital Multimeter. Additionally, the RC-time constant of the circuit (including the RTD) was experimentally determined to be $\tau_{R C} \cong 250 \mathrm{~ns}$ across the applied voltage range. Hence, $\tau_{R C}$ is orders of magnitude below the lifetime of photoinduced holes and thus can be neglected. Fig. 3(a) displays the photocurrent in a colored contour plot as a function of bias voltage and time. Dark blue represents no photocurrent and dark red the highest measured photocurrent of $160 \mathrm{nA}$.

Fig. 3(b) shows temporal traces of $I_{P h}$ for three different bias voltages: $1.50 \mathrm{~V}$ (black line), $2.00 \mathrm{~V}$ (red line), and $2.50 \mathrm{~V}$ (green line). When the light pulse is switched on, the photocurrent rises exponentially, and when the light pulse is switched off again, an exponential decline can be observed. Rise and decay are fastest for the highest bias voltage $(V=2.50 \mathrm{~V})$ and slowest for $V=1.50 \mathrm{~V}$. Also, the rise and decay of $I_{P h}(t)$ are not purely mono-exponential. There are several possible explanations for the non mono-exponential rise and decay, such as separate populations with different escape channels, a mean lifetime $\tau\left(V, N_{h}\right)$ that is also a function of accumulated charge carriers, or a combination of both. We have determined $\tau$ by taking the time at which $I_{P h}(t)$ has decreased to $1 / e$ of its maximum.
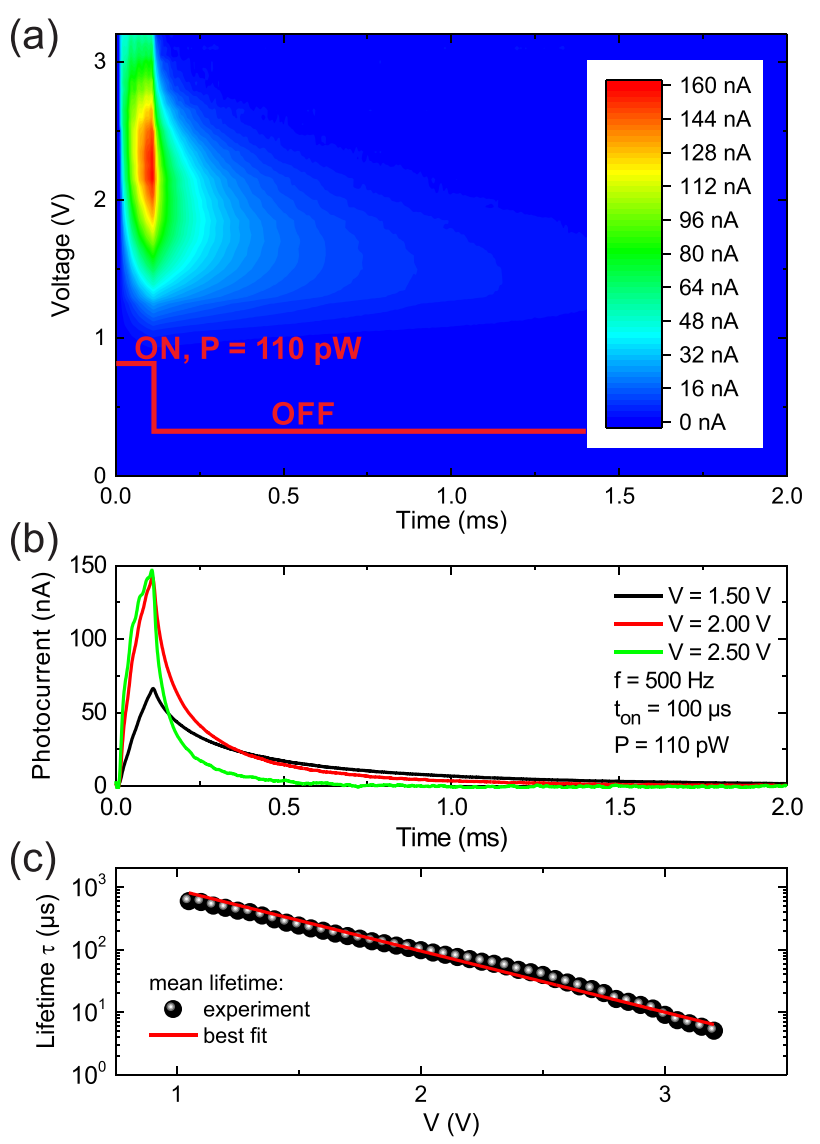

FIG. 3. (a) Colored contour plot of the RTD photocurrent as a function of bias voltage and time. Red represents the highest measured photocurrent of $180 \mathrm{nA}$ and dark blue no photocurrent. (b) Photocurrent versus time for three different applied bias voltages. (c) Mean lifetime of photogenerated holes accumulated at the resonant tunneling structure versus bias voltage. 
The mean lifetime of holes is plotted on the logarithmic scale versus bias voltage in Fig. 3(c). Over the whole voltage range, $\tau$ is found to decrease exponentially with $V$ from $\tau=597 \mu$ s at $1.05 \mathrm{~V}$ to $\tau=5 \mu \mathrm{s}$ at $3.20 \mathrm{~V}$. This compares well with the values in the order of $100 \mu$ s that have been reported for a similar RTS at cryogenic temperatures. ${ }^{9}$ Nevertheless, considering the room temperature environment of our experiment, the measured lifetimes seem strikingly high, since with increasing temperature, further escape channels such as thermionic emission are triggered in addition to pure tunneling. Indeed, the exponential decay of $\tau$ with $V$ suggests a thermal process to be the dominating factor in the escape of accumulated holes. For our sample, we propose a simple exponential decay

$$
\tau(V)=\tau_{0} \times \exp \left(-\frac{q_{0} V}{\beta \times k_{B} T}\right),
$$

where $\tau_{0}$ is the lifetime for zero bias, $k_{B}$ is the Boltzmann's constant, $q_{0}$ is the elementary charge, $T$ is the temperature, and $\beta$ is a dimensionless decay constant. Using Eq. (5) as fitting equation, we find $\tau_{0}=8.65 \mathrm{~ms}$ and $\beta=17.3$.

By inserting Eqs. (5) and (6) into Eq. (4), $\Delta V(V)$ can be calculated as

$$
\begin{aligned}
\Delta V(V)= & \frac{\eta_{0}}{2}\left(1+\operatorname{erf}\left(\gamma\left(V-V_{t h, \eta}\right)\right)\right) \times \tau_{0} \\
& \times \exp \left(-\frac{q_{0} V}{\beta \times k_{B} T}\right) \times \frac{P}{h \nu} \times \frac{V_{h}}{n_{R T D}} .
\end{aligned}
$$

Then, $I_{P h}(V)$ can be calculated by inserting Eq. (7) into Eq. (1). Indeed, this set of equations provides useful insight on how to optimize RTD photodetectors.

In Fig. 4(a), the photoinduced voltage shift $\Delta V$ (black line) according to Eq. (7) is shown schematically as a

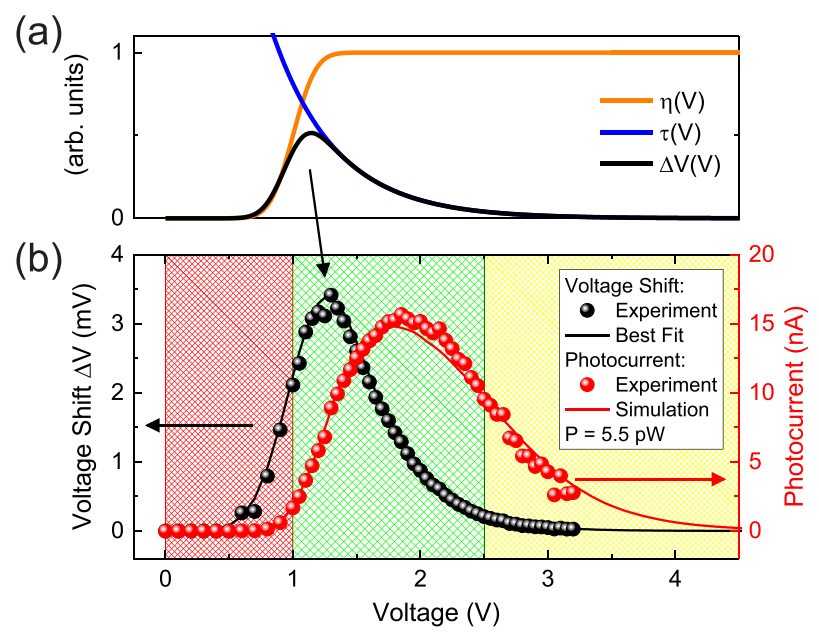

FIG. 4. (a) Scheme of the RTD's quantum efficiency $\eta(V)$ (orange line) and the mean lifetime $\tau(V)$ (blue line) of accumulated holes as a function of bias voltage. The product of $\eta(V)$ and $\tau(V)$ determines the RTD's photoresponse $\Delta V(V)$ (black line). (b) Experimentally determined values (black spheres) and best fit (black line) of the photoinduced voltage shift at an illumination power of $P=5.5 \mathrm{pW}$. Additionally shown are the corresponding experimental values (red spheres) and the simulation (red line) of the photocurrent. The bias voltage region with the highest photoresponse is shaded green. The poorest photoresponse is shaded red. The yellow shaded region provides low photoresponse with high cut-off frequencies. function of bias voltage $V . \Delta V$ is governed by the RTD's quantum efficiency $\eta(V)$ at low voltages and by the lifetime of accumulated holes $\tau(V)$ (blue line) for higher voltages.

Experimentally determined values (black spheres) and best fit after Eq. (7) (black line) of the photoinduced voltage shift at an incident power of $5.5 \mathrm{pW}$ are shown in Fig. 4(b). The corresponding photocurrent is shown as red spheres (experimental data) and red line (simulation). For the experimental data, we extracted the following parameters: $\left(\left(\eta_{0} \tau_{0} V_{h}\right) /\left(2 n_{R T D} h \nu\right)\right)=1.60 \times 10^{10} \mathrm{~V} / \mathrm{W}, \gamma=2.87, V_{t h, \eta}$ $=1.22 \mathrm{~V}$, and $\beta=14.4$. While $\gamma$ and $V_{t h, \eta}$ are in excellent agreement with the expected values (due to the Gaussian fit, see Fig. 2(f)), $\beta$ is slightly reduced with respect to the expected value of 17.3. The best fit value for $\left(\left(\eta_{0} \tau_{0} V_{h}\right) /\left(2 n_{R T D} h \nu\right)\right)$ is reduced by a factor of 3.07. The most likely explanation for such a discrepancy is a more realistic maximum RTD quantum efficiency of $\eta_{0}=1.7 \%$, since the estimated value of $5.2 \%$ was calculated without considering additional loss mechanisms that might take place. One can distinguish three different voltage regions for RTD light detection. The red shaded region of up to $1.00 \mathrm{~V}$ marks the worst possible choice for setting the operation point. Here, the RTD's quantum efficiency is close to zero, which suppresses any photosensitivity. The green shaded region from $1.00 \mathrm{~V}$ up to $2.50 \mathrm{~V}$ represents the region of highest photosensitivity, with a maximum voltage shift and photocurrent. The yellow shaded region for voltages greater than $2.50 \mathrm{~V}$ is governed by decreasing lifetimes of accumulated holes and the photoresponse decreases. However, reduced mean lifetimes lead to faster response times, which qualifies this region for higher operating frequencies.

In summary, we have presented a general study of the RTD photoresponse and the photocurrent-voltage relation of an RTD photodetector. The discussion is applicable to any kind of RTD photodetector and leaves room for further modifications by adjusting Eqs. (5) and (6). The photoresponse of an RTD is defined by three basic and voltage dependent parameters: (i) The quantum efficiency $\eta(V)$, which is a measure of how efficiently photons excite carriers that are then captured for accumulation at the RTS. (ii) The mean lifetime of accumulated charge carriers at the RTS. (iii) Electronic transport properties of the RTS, by name, the RTD's current-voltage characteristics in the dark. The most crucial and limiting parameter is the lifetime of photogenerated and accumulated charge carriers, which sets upper bounds for the device sensitivity and operation frequency.

The authors are grateful for financial support by the BMBF via national project EIPHRIK (FKZ: 13N10710), the European Union (FPVII (2007-2013) under Grant Agreement No. 318287 LANDAUER), and the Brazilian Agencies FAPESP (2012/51415-3 and 2012/13052-6), CNPq and CAPES. The expert technical assistance by $\mathrm{M}$. Emmerling and S. Handel is gratefully acknowledged.

\footnotetext{
${ }^{1}$ R. Tsu and L. Esaki, Appl. Phys. Lett. 22, 562 (1973).

${ }^{2}$ L. L. Chang, L. Esaki, and R. Tsu, Appl. Phys. Lett. 24, 593 (1974).

${ }^{3}$ M. Feiginov, C. Sydlo, O. Cojocari, and P. Meissner, Appl. Phys. Lett. 99, 233506 (2011).

${ }^{4}$ M. Feiginov, H. Kanaya, S. Suzuki, and M. Asada, Appl. Phys. Lett. 104, 243509 (2014).
} 
${ }^{5}$ P. Mazumder, S. Kulkarni, M. Bhattacharya, and G. I. Haddad, Proc. IEEE 86, 664 (1998).

${ }^{6}$ A. Pfenning, F. Hartmann, M. Rebello Sousa Dias, L. K. Castelano, C. Süßmeier, F. Langer, S. Höfling, M. Kamp, G. E. Marques, L. Worschech, and V. Lopez-Richard, ACS Nano 9, 6271 (2015).

${ }^{7}$ A. Pfenning, F. Hartmann, F. Langer, S. Höfling, M. Kamp, and L. Worschech, Appl. Phys. Lett. 104, 101109 (2014).

${ }^{8}$ G. Haddad, P. Mazumder, and J. N. Schulman, Proc. IEEE 86, 641 (1998).

${ }^{9}$ J. Blakesley, P. See, A. Shields, B. Kardynat, P. Atkinson, I. Farrer, and D. Ritchie, Phys. Rev. Lett. 94, 067401 (2005).

${ }^{10}$ S. S. Hees, B. E. Kardynal, P. See, A. J. Shields, I. Farrer, and D. A. Ritchie, Appl. Phys. Lett. 89, 153510 (2006).

${ }^{11}$ H. W. Li, B. E. Kardynał, P. See, A. J. Shields, P. Simmonds, H. E. Beere, and D. A. Ritchie, Appl. Phys. Lett. 91, 073516 (2007).

${ }^{12}$ W. Wang, Y. Hou, D. Xiong, N. Li, W. Lu, W. Wang, H. Chen, J. Zhou, E. Wu, and H. Zeng, Appl. Phys. Lett. 92, 023508 (2008).
${ }^{13}$ R. H. Hadfield, Nat. Photonics 3, 696 (2009).

${ }^{14}$ M. A. Rowe, E. J. Gansen, M. Greene, R. H. Hadfield, T. E. Harvey, M. Y. Su, S. W. Nam, R. P. Mirin, and D. Rosenberg, Appl. Phys. Lett. 89, 253505 (2006).

${ }^{15}$ P. W. Park, H. Y. Chu, S. G. Han, Y. W. Choi, G. Kim, and E.-H. Lee, Appl. Phys. Lett. 67, 1241 (1995).

${ }^{16}$ I. J. S. Coêlho, J. F. Martins-Filho, J. M. L. Figueiredo, and C. N. Ironside, J. Appl. Phys. 95, 8258 (2004).

${ }^{17}$ F. Hartmann, F. Langer, D. Bisping, A. Musterer, S. Höfling, M. Kamp, A. Forchel, and L. Worschech, Appl. Phys. Lett. 100, 172113 (2012).

${ }^{18}$ P. England, J. E. Golub, L. T. Florez, and J. P. Harbison, Appl. Phys. Lett. 58, 887 (1991).

${ }^{19}$ I. Camps, A. Vercik, L. F. dos Santos, and Y. G. Gobato, Brazilian J. Phys. 36, 343 (2006).

${ }^{20}$ J. Geisz, D. Friedman, J. Olson, S. R. Kurtz, and B. M. Keyes, J. Cryst. Growth 195, 401 (1998). 